УДК 512.552 .13

B. V. Zabavsky, O. M. Romaniv

\title{
RINGS WITH THE KAZIMIRSKY CONDITION AND RINGS WITH PROJECTIVE SOCLE
}

\begin{abstract}
B. V. Zabavsky, O. M. Romaniv. Rings with the Kazimirsky condition and rings with projective socle, Mat. Stud. 51 (2019), 124-129.

We construct the theory of diagonalizability for matrices over Bezout rings of stable range 1 with the Kazimirsky condition. It is shown that a ring of stable range 1 with the right (left) Kazimirsky condition is an elementary divisor ring if and only if it is a duo ring. We describe the conditions under which a proper finite homomorphic image of a commutative Bezout domain is a ring with projective socle.
\end{abstract}

1. General Appearance. Elementary divisor rings have been studied by many authors. The most full history of this rings can be found in [16]. Recall by I. Kaplansky: a ring $R$ is called an elementary divisor ring if for an arbitrary matrix $A$ over $R$ there exist invertible matrices $P$ and $Q$ of suitable sizes such that $P A Q=D$ is a diagonal matrix $D=\left(d_{i}\right)$ and $R d_{i+1} R \subseteq R d_{i} \cap d_{i} R$ for each $i([9])$. In the case of commutative rings there are many developments on this rings, in the case of noncommutative rings they are little investigated and fragmented. A general picture is far from its full description. Among these results one should especially note the result [15] which shows that a distributive elementary divisor domain is duo. Tuganbaev extended this result in case of a distributive ring ([14]).

In [8] Henriksen proved that if $R$ is a unit regular ring than every matrix over $R$ admits diagonal reduction. Let us note that a regular ring is a unit regular if and only if this ring is a ring of stable range 1 . Recall, that an associative $\operatorname{ring} R$ with identity $(1 \neq 0)$ is a ring of stable range 1 if for any $a, b \in R$ such that $a R+b R=R$ there exists $t \in R$ such that $(a+b t) R=R([3])$. It is an open problem: when a ring of stable range 1 is an elementary divisor ring?

In the case of commutative rings Kaplansky announced that a commutative ring of stable range 1 is an elementary divisor ring if and only if it is a Bezout ring (by a Bezout ring we mean a ring in which all finitely generated ideals are principal).

An important role in studying the elementary divisor rings are played by the Hermite rings. A ring is called a right (left) Hermite ring if all $1 \times 2(2 \times 1)$ matrices have diagonal reduction, i.e. a ring $R$ is called a right (left) Hermite ring if for any $a, b \in R$ there exist invertible matrices $P$ and $Q$ such that $(a, b) P=(d, 0)\left(Q\left(\begin{array}{l}a \\ b\end{array}\right)=\left(\begin{array}{l}c \\ 0\end{array}\right)\right)$ for some elements $d, c \in R$. An Hermite ring is a ring which is both right and left Hermite ring.

2010 Mathematics Subject Classification:06F20, 13 F99.

Keywords: Bezout ring; Kazimirsky condition; elementary divisor ring; stable range; unit stable range; projective socle; maximal ideal; Kasch ring; $P S$-ring; $P S$-module; unit-central ring; duo ring. doi:10.15330/ms.51.2.124-129

(C) B. V. Zabavsky, O. M. Romaniv, 2019 
For a ring the property of being Hermite plays an important role in considering the possibility of diagonal reduction of matrices, since this condition is present in all known necessary and sufficient conditions of theorems on elementary divisor rings.

Note that any Hermite ring is a finitely generated principal ideal ring, i.e. a ring in which an arbitrary finitely generated right or left ideal is principal (in modern terminology these rings are called Bezout rings). You should not think that an arbitrary Bezout ring of stable range 1 is an elementary divisor ring. N. I. Dubrovin ([5]) showed that any semilocal and semiprimary Bezout ring $R$ is an elementary divisor ring if and only if for any element $a \in R$ there exists $b \in R$ such that $R a R=b R=R b$. Note that a semilocal ring is a ring of stable range 1 and also note that a right (left) Bezout ring of stable range 1 is a right (left) Hermite ring $([3])$.

In modern research on the theory of rings a peculiar place is occupied by the rings in which every invertible element lies in the center of the ring, i.e. unit-central rings ([10]). These same authors raised an open question: it is every unit-central ring with stable range 1 commutative?

Finite homomorphic images of a commutative Bezout domain are sources of examples and counterexamples in the current research in the ring theory $([2,4,6,7,12,13,17])$. In this article we describe the condition under which a proper finite homomorphic images of a commutative Bezout domain are rings with projective socle. A study of rings with projective socle was made by R. Gordon ([7]). More recently G. Baccella and W. K. Nicholson with J. F. Watters have provided a number of necessary and sufficient conditions on rings for rings to have a projective socle $([1,12])$.

2. Diagonal reduction of matrices over Bezout rings of stable range 1 with the Kazimirsky condition. In this section we consider Bezout rings of stable range 1 with Kazimirsky condition (which are generalizations unit-central rings).

Let $R$ be an associative ring with identity $(1 \neq 0)$.

Definition 1. A ring $R$ is a ring with the right (left) Kazimirsky condition if for any $a \in R$ and any invertible element $u \in R$ the following inclusion holds $a R \supseteq u a R(R a \supseteq R a u)$. If $R$ is a ring with the right and left Kazimirsky condition then we say, that $R$ is a ring with the Kazimirsky condition.

Obviously, an unit-central ring is a ring with the Kazimirsky condition.

Proposition 1. Let $R$ be a ring of stable range 1. Then $R a=R a u$ for any $a \in R$ and any invertible $u \in R$ if and only if $a u=v a$ for some invertible $v \in R$.

Proof. If $a u=v a$ for some invertible $v \in R$, then obviously $R a u=R a$.

Let $R a=R a u$. Since $R$ is a ring of stable range 1, by [16] we have $v a=a u$ for some invertible $v \in R$.

Proposition 2. Let for any $a \in R$ and any invertible element $u \in R$ there exists $x \in R$ such that $1+x a=u$. Then $R a u \subseteq R a$ for any invertible $u \in R$ and any $a \in R$.

Proof. Since $1+x a=u$, we have $a+a x a=a u$ and $a u \in R a$, i.e. $R a u \subseteq R a$.

Theorem 1. Let $R$ be a ring of stable range 1 with the left Kazimirsky condition. Then for any $a, b \in R$ if $a R+b R=R$, then $R a+R b=R$. 
Proof. Let $a R+b R=R$. Since $R$ is a ring of stable range $1, a+b t=u$ for some $t \in R$ and an invertible $u \in R$. Then $R a+R t=R$, i.e. $a x+t=w$ for some $x \in R$ and an invertible $w \in R$. The equality $t=w-x a$ implies $u=a+b(w-x a)=a+b w-b x a=(a-b x) a+b w$. Since $R$ is a ring with the left Kazimirsky condition we have $R b w \subseteq R b$, i.e. $b w=y b$ for some $y \in R$. Thus $u=(a-b x) a+y b$, i.e. $R a+R b=R$.

Recall that a ring $R$ is a right (left) quasi-duo ring if any right (left) maximal ideal is an ideal. A right and left quasi-duo ring is called a quasi-duo ring. By Theorem 1 we have the following result.

Proposition 3. A ring of stable range 1 with the left (right) Kazimirsky condition is a left (right) quasi-duo ring.

Proof. Let $M$ be an arbitrary left maximal ideal. If $M$ is not two-sided then $m r \notin M$ for some $m \in M$ and $r \in R$. Therefore, $M+R m r=R$ and hence $n+x m r=1$ for some $n \in M$ and $x \in R$. By Theorem 1 we have $R n+R x m=R$.

Since $m \in M$, we have $x m \in M$, i.e. $R n+R x m \subseteq M$. This is, of course, impossible, so $M$ must be a two-sided ideal. By [15] the proposition is proved.

According to results of $[14,15]$ we have the following result.

Theorem 2. A Bezout ring of stable range 1 with the Kazimirsky condition is an elementary divisor ring if and only if it is a duo ring.

Note that a duo ring is a ring with the Kazimirsky condition. In fact, if $R$ is a duo ring and for any $a \in R$ and any invertible $u \in R$ we have $a u=x a(u a=a y)$ for some $x, y \in R$, i.e. $R a u \subseteq R a(u a R \subseteq a R)$.

Recall that a ring $R$ is said to have the unit stable range 1 if $a R+b R=R$ implies that there exists an invertible $u \in R$ such that $(a+b u) R=R$.

We note that a regular ring $R$ has the unit stable range 1 if and only if it is unit-regular and for any idempotent $e, f \in R, e R+f R=R$ implies that there exist invertible $u, v \in R$ such that $e u+f v=1$. Moreover, $M_{2}\left(\mathbb{Z}_{2}\right)$ has the unit stable range 1 , while $\mathbb{Z}_{2}$ does not have the unit stable range $1[3]$.

A ring of unit stable range 1 is a ring in which any additively generated by its units.

Proposition 4. Let $R$ be a ring of unit stable range 1. Thyn for any nonzero $a \in R$ we have $a=u+w$ for some invertible $u, w \in R$.

Proof. Let $a \in R \backslash\{0\}$. Then $a R+(-1) R=R$. Since $R$ is a ring of unit stable range 1 then exists an invertible $u \in R$ such that $a-u=w$ is an invertible element of $R$, i.e. $a=u+w$.

Proposition 5. A ring of unit stable range 1 is a ring with the right (left) Kazimirsky condition if and only if it is a left (right) duo ring.

Proof. Let $R$ be a ring of unit stable range 1, and a ring with right Kazimirsky condition and let $a, b \in R$. By Proposition 4, we have $a=u+w$ for some invertible $u, w \in R$. Since $R$ is a ring with the right Kazimirsky condition we have $u b R \subseteq b R$ and $w b R \subseteq b R$, i.e. $u b=b x$ and $w b=b y$ for some $x, y \in R$. Therefore $a b=(u+w) b=u b+w b=b x+b y=b(x+y)$, i.e. $R b \subseteq b R$, and $R$ is a left duo ring.

From these results we have the following consequence. 
Theorem 3. A Bezout ring of unit stable range 1 with the Kazimirsky condition is an elementary divisor ring.

Example. In the paper [11] it was proved that if the skew polynomial ring $R[x ; \sigma]$ is left or right duo, then $R[x ; \sigma]$ is commutative.

Let $R=\mathbb{Z}[\sqrt{-7}]$ and $K=\mathbb{Q}(\sqrt{-7})$ be the quotient field of the ring $R$. We consider an automorphism $\sigma: K \rightarrow K$ such that $\sigma(a+b \sqrt{-7})=a-b \sqrt{-7}, a, b \in \mathbb{Q}$. We then consider the subring $S$ of the ring $K[x ; \sigma]$ which consists of polynomials the free member of which belongs $R$. The only units in the ring $S$ are 1 and -1 , therefore the ring $S$ is a unit-central ring but not commutative.

3. When finite homomorphic images of a commutative Bezout domain are rings with projective socle? Throughout the section, all the rings $R$ will be commutative rings and have a unity, and all the modules will be unitary. By $U(R)$ we denote the group of units of $R$. The annihilator of a subset $X$ of a ring $R$ is denoted by $X^{\perp}$. By $J(R)$ we denote the Jacobson radical of a ring $R$.

First note the following results.

Proposition 6. Let $R$ be a commutative Bezout domain and $a \in R \backslash\{0\}$ such that there exists a decomposition $a=b c$, where $b, c \notin U(R)$ and $b R+c R \neq R$. Then $J(R / a R) \neq\{\overline{0}\}$.

Proof. Since $a=b c$, where $b, c \notin U(R)$ and $b R+c R=d R, d \notin U(R)$, we deduce $b=d b_{0}$, $c=d c_{0}$, where $b_{0} R+c_{0} R=R$. Consider the element $k=b c_{0}$. Obviously $\bar{k}=k+a R$ is a nonzero element of $R / a R$ and $\bar{k}^{2}=\overline{0}$. Since $k^{2}=b c_{0} b c_{0}=b c b_{0} c_{0} \in a R$. We have $\bar{k} \in J(R / a R)$.

Proposition 7. Let $R$ be a commutative Bezout domain and $a \in R \backslash\{0\}$. Then $J(R / a R)=$ $\{0\}$ if and only if for any decomposition $a=b c$, where $b, c \notin U(R)$ we have $b R+c R=R$.

Proof. According to Proposition 6 we have to prove sufficiency.

Let for any decomposition $a=b c$, where $b, c \notin U(R)$ we have $b R+c R=R$. Let $\bar{d} \in$ $J(R / a R)$ and $\bar{d} \neq \overline{0}$. Denote $\bar{d}=d+a r$ and consider $d R+a R=k R$. Notice that if $k \in U(R)$ then $\bar{d} \in U(R / a R)$. Since $k \notin U(R)$, we have $d=k d_{0}, a=k a_{0}$ and $d_{0} R+a_{0} R=R$ for some $a_{0}, d_{0} \in R$. Notice that if $a_{0} \in U(R)$, then $d \in a R$, i.e. $\bar{d}=\overline{0}$. We have that $a=a_{0} k$, where $a_{0} \notin U(R), k \notin U(R)$ and $a_{0} R+k R=R$, i.e. $a_{0} x+k y=1$ for some $x, y \in R$. Since $d R+a R=k R$ and $\bar{d} \in J(R / a R)$. Since $a_{0} x+k y=1$, we have $\overline{a_{0}} \bar{x}+\bar{k} \bar{y}=\overline{1}$, i.e. $\overline{a_{0}} \bar{x} \in U(R / a R)$, i.e. $a_{0} R+a R=R$, i.e. $a_{0} \in U(R)$. This contradiction proves our results.

Definition 2. An ideal $I$ of a ring $R$ is called $\mathrm{X}$ dense if $I^{\perp}=\{0\}$. A commutative ring in which any maximal ideal is not dense is called a Kasch ring.

The following theorem gives examples of Kasch rings.

Theorem 4 ([17], Theorem 3). Let $R$ be a commutative Bezout domain and a be some nonzero element $R$. The following statement are equivalent:

1) $R / a R$ is a Kasch ring;

2) any maximal ideal that contains the element $a$ is principal. 
Proposition 8. Let $R$ be a commutative ring and $(J(R))^{\perp}=\{0\}$. Then every maximal ideal of the ring $R$ is dense.

Proof. For every maximal ideal $M$ we have $J(R) \subset M$. Since $M^{\perp} \subset(J(R))^{\perp}$ we have that $M$ is dense.

Rings $R$ in which $\left((J(R))^{\perp}=(0)\right.$ are examples of $P S$-rings (rings with projective socle).

Definition 3. A right $R$-module $M_{R}$ is called a $P S$-module if its every simple submodule is projective, equivalently, if $\operatorname{soc}\left(M_{R}\right)$ is projective. A ring $R$ is called a $P S$-ring if $R_{R}$ is a PS-module ([12]).

Theorem 5. Let $R$ be a commutative $P S$-ring which is a Kasch ring. Then the ring $R$ is a finite direct sum of fields.

Proof. Since $R$ is a commutative $P S$-ring, by [12], Theorem 2.4, then for an ideal $M$ we have $M=(0)$ or $M=e R$, where $e^{2}=e \in R$. Because $R$ is a Kasch ring, we have that for any maximal ideal $M$ there exists an idempotent $e^{2}=e \in R$ such that $M=e R$. By [13], Theorem 3.2, we have that $R$ is a finite direct sum of fields.

As a consequence we obtain the main result of the article.

Theorem 6. Let $R$ be a commutative Bezout domain and a be a nonzero element which is contained only in a principal maximal ideal $m_{i} R$. Then $R / a R$ is a $P S$-ring if and only if $a$ is contained only in a finite set of maximal ideals $m_{1} R, m_{2} R, \ldots, m_{n} R$ and $a=m_{1} m_{2} \ldots m_{n}$.

Proof. By Theorem 4 we have that $R / a R$ is a Kasch ring. By Theorem 5 we have that $R / a R$ is a finite direct sum of fields. Obviously $R / a R$ is a semilocal ring, i.e. $a$ is contained in a finite set of maximal ideals. Since $J(R / a R)=(0)$, by Proposition 7 we have that for any decomposition $a=b c$ where $b, c \notin U(R)$ we have $b R+c R=R$.

Let $M_{1}=m_{1} R, m_{2} R, \ldots, M_{n}=m_{n} R$ be all the maximal ideals of $R$ which contain the element $a$. Then $a=m_{1} b_{1}$, where $b_{1} \notin m_{1} R$, i.e. $a=m_{1} m_{2} b_{2}$ where $b_{2} \notin m_{1} R, b_{2} \notin m_{2} R$, then we have $a=m_{1} m_{2} \ldots m_{n}$.

Now let it be $a=m_{1} m_{2} \ldots m_{n}$ where $m_{1} R, m_{2} R, \ldots, m_{n} R$ are all the maximal ideals of $R$ which contain the element $a$.

Since

$$
R / a R \simeq R / m_{1} R \oplus R / m_{2} R \oplus \cdots \oplus R / m_{n} R
$$

we have that $R / a R$ is a finite direct sum of fields. By Theorem 3.2 [13] we have that any maximal ideal of $R / a R$ is generated by an idempotent. By Theorem 2.4 [12] we have that $R / a R$ is a $P S$-ring.

\section{REFERENCES}

1. G. Baccella, On \&-semisimple rings. A study of the socle of a ring, Comm. Alg., 8 (1980), №10, 889-909.

2. V. Camillo, W.K. Nicholson, Z. Wang, Left quasi-morphic rings, J. Alg. Appl., 7 (2008), №6, 725-733.

3. H. Chen, Rings related to stable range conditions Series in Algebra 11, World Scientific, Hackensack, NJ, 2011. 
4. R.R. Colby, Rings which have flat injective modules, J. Alg., 35 (1975) 239-252.

5. N.I. Dubrovin, On noncommutative rings with elementary divisors, Reports of Institutes of Higher Education Math., 11 (1986), 14-20.

6. G.A. Garkusha, FP-injective and weakly quasi-Frobenius rings, J. Math. Scien., 112 (2002), №3, 43034312.

7. R. Gordon, Rings in which minimal left ideals are projective, Pacific J. Math., 31 (1969), №3, 679-692.

8. M. Henriksen, On a class of regular rings that are elementary divisor rings, Archiv Math., 24 (1973), №1, 133-141.

9. I. Kaplansky, Elementary divisors and modules, Trans. Amer. Math. Soc., 66 (1949) 464-491.

10. D. Khurana, G. Marks, A.K. Srivastava, On unit-central rings, Springer, Advances in Ring Theory, Trends in Mathematics, Birkhäuser Verlag-Basel/Switzerland, 2010, 205-212.

11. G. Marks, Duo rings and Ore extensions, J. Alg., 280 (2004), №2, 463-471.

12. W.K. Nicholson, J.F. Watters, Rings with projective socle, Proc. Amer. Math. Soc., 102 (1988), №3, 443-450.

13. M. Satyanarayana, Rings with primary ideals as maximal ideals, Math. Scand., 20 (1967), 52-54.

14. A.A. Tuganbaev, Rings of elementary divisors and distributive rings, Russ. Math. Surv., 46 (1991), №6, 219-220.

15. B.V. Zabavsky, M.Ya. Komarnytsky, Distributive elementary divisor domains, Ukr. Math. J., 42 (1990), №7, 890-892.

16. B. V. Zabavsky, Diagonal reduction of matrices over rings, Mathematical Studies, Monograph Series, V.XVI, VNTL Publishers, Lviv, 2012.

17. B. Zabavsky, O. Pihura, Bezout morphic rings, Visnyk Lviv Univ., 79 (2014), 163-168.

Department of Mechanics and Mathematics

Ivan Franko National University

Lviv, Ukraine

zabavskii@gmail.com

oleh.romaniv@lnu.edu.ua 